\title{
Correlation Between Tumor Necrosis Factor Alpha and Proteinuria in Type-2 Diabetic Patients
}

\author{
Hany A. Refaat, Gamal E. Mady, Mohammed M. Abd El Ghany, Khaled H. Abou Seif, Eman S. El \\ Hadidi, Yasser Elshahawy*, Dawlat Sany and Haitham E. Abd El Aziz
}

Division of the renal disease, Ain shams universty

\begin{abstract}
Introduction: Diabetic Nephropathy (DN) is the single most common cause of end stage renal disease (ESRD) in many countries. Inflammation is a potential factor in the development and progression of $\mathrm{DN}$ and recent data indicate that diabetes includes an inflammatory component which may contribute to diabetic complications.
\end{abstract}

Methods: This study was conducted at Ain Shams University Hospital on 95 patients with type-2 diabetes mellitus complicated with retinopathy and fifteen ageand sex-matched healthy volunteers. Diabetic patients were divided into 4 groups according to the degree of proteinuria. Serum tumor necrosis factor- $\alpha$ (TNF- $\alpha)$, urine TNF- $\alpha$ and C-reactive protein (CRP) levels were measured in all subjects. Correlations between these inflammatory parameters and degree of proteinuria, duration of diabetes and degree of glycemic control were examined.

Results: Levels of the three inflammatory parameters were significantly higher in diabetic patients when compared to control subjects, and they were positively correlated to urinary protein excretion. There was significant positive correlation between serum and urine TNF- $\alpha$ and duration of diabetes, as well as between serum TNF- $\alpha$ and glycemic control. Serum and urine TNF- $\alpha$ remained as independent predictors of urine protein excretion in diabetic patients with overt proteinuria after forward stepwise multiple regression analysis.

Conclusion: Serum and urine TNF- $\alpha$ and CRP levels are significantly elevated in this group of diabetic patients, and correlate positively with severity of proteinuria. This suggests a significant role for TNF- $\alpha$ in the pathogenesis and progression of renal injury in diabetes mellitus.

\footnotetext{
* Corresponding author; Ain Shams University, Division of Rena Disease, Faculty of Medicine, Abbasia square, Cairo, Egypt.

E mail: yasserelshahawi@yahoo.com
}

Keywards: Diabetic nephropathy; Proteinuria; Tumor necrosis factor- $\alpha$

\section{The authors declared no conflict of interest}

\section{Introduction}

Diabetic Nephropathy (DN) is the single most common cause of end stage renal disease (ESRD) in many countries. It accounts for more than $20 \%$ of the cases of chronic renal failure [1]. The pathogenic mechanisms and molecular events involved in the development and progression of DN are not completely known. Traditionally, the development of renal lesions in patients with type 2 diabetes mellitus is attributed to interactions of metabolic and hemodynamic factors. Inflammation is a potential factor in the development and progression of $\mathrm{DN}$, and recent data indicate that an inflammatory component contributes to the development of diabetic complications [2-6].

Tumor Necrosis Factor alpha (TNF- $\alpha$ ) is a 157 amino-acid peptide produced mainly by monocytes, macrophages, B and T lymphocytes as well as glomerular mesangial cells. Elevated TNF- $\alpha$ level in urine is found and accused to play a role in some immune mediated glomerulonephritidis (e.g. IgA nephropathy and AntiGlomerular Basement Membrane Disease) [7]. The role of TNF- $\alpha$ in mediating renal and glomerular lesions was not elucidated until 1989, when Bertani and associates observed that the infusion of human recombinant TNF- $\alpha$ into rabbits induced the presence of inflammatory cells in the glomerular capillaries, with the prominent findings of glomerular endothelial damage, polymorphonuclear cell accumulation and fibrin deposition within the capillary lumen [8]. Since then, diverse studies have demonstrated that this cytokine, through a broad series of bioactivities, may promote significant actions in the kidney with the development of renal injury [9]. 
The local actions and implications of intra-renal TNF- $\alpha$ are interesting. In 1991, Hasegawa et al reported that macrophages incubated with glomerular basement membranes from diabetic rats produced greater amounts of TNF- $\alpha$ than did macrophages incubated with membranes from non-diabetic animals [10]. These authors suggested for the first time the potential implication of this cytokine in the pathogenesis of DN. Furthermore, TNF- $\alpha$ is a potential causative agent implicated in glomerular and tubulointerstitial damage in the setting of diabetes [11]. Also, a recent study by Moriwaki et al found that TNF- $\alpha$ inhibition is a potential therapeutic strategy in experimental diabetic rats [12]. It is important to note that this cytokine may be produced in the diabetic kidney by infiltrating cells (mainly macrophages) as well as intrinsically by renal cells (endothelial, mesangial, glomerular and tubular epithelial cells) [4]. Within the glomerulus, TNF- $\alpha$ displays a number of effects that are relevant to the manifestations observed at the initial stages and during the progression of glomerular injury. TNF- $\alpha$ is implicated in the misbalance between vasodilator and vasoconstrictor mediators, which may result in alterations of glomerular blood flow and glomerular filtration rate. Additionally, this cytokine is cytotoxic to glomerular, mesangial and epithelial cells, and may induce direct renal damage. Also TNF- $\alpha$ is able to promote the local generation of reactive oxygen species, which affects the barrier function of the glomerular capillary wall resulting in enhanced albumin permeability, independently of hemodynamic factors or effects of recruited inflammatory cells. Regarding tubulointerstitial damage, exposure of tubular epithelial cells to TNF- $\alpha$ increased the synthesis and secretion of lymphocyte and neutrophil chemoattractant factors as well as the cell surface expression of intercellular adhesion molecule-1, which has been implicated in the development of renal injury in diabetes. Finally, TNF- $\alpha$ has stimulatory effects on sodium uptake by proximal tubule cells, contributing to sodium retention and renal hypertrophy, typical alterations that occur during the early stage of DN [3].

\section{Methods}

This cross-sectional study was conducted on 95 type-2 diabetic patients with retinopathy and 15 healthy control subjects. Diabetic patients with retinopathy were divided into 4 groups: Group-I included twenty diabetic patients with microalbuminuria and estimated GFR $>90$, group-2 included thirty patients with overt albuminuria and estimated GFR >90, group-III included thirty patients with overt albuminuria and estimated GFR $<30$, and group-IV included fifteen diabetic patients without albuminuria and with estimated GFR $>90$.
To avoid potential confounding factors, we excluded patients with current acute illness (including infections), current cigarette smoking, immunologic or malignant diseases, morbid cardiovascular illness, medical history of clinical cardiovascular events (cardiac, cerebral or peripheral vascular disease) in the last 6 months, and patients on angiotensin converting enzyme inhibitors (ACEi), angiotensin receptor antagonists or nonsteroidal anti-inflammatory drugs (NSAIDs). Before inclusion, the possible existence of active immunological diseases, malignancy or infections was investigated if suspected. White blood cell count was lower than 10000/ $\mathrm{mm}^{3}$ in all cases. Tumor markers including carcinoembryonic antigen, $\alpha$-fetoprotein, cancer antigen- 125 and prostate-specific antigen were negative in screened subjects. Serological tests for antinuclear antibodies, antineutrophilic cytoplasmic antibodies, cryoglobulins, rheumatoid factor and complement factors C3 and C4 were negative or within the normal range. Urine cultures and serology for hepatitis B and C and human immunodeficiency virus were also negative.

All subjects gave informed consent to participate in the study. Relevant clinical history and examination were recorded. The presence of diabetic nephropathy was an inclusion criterion for all diabetic patients in the study. Arterial blood pressure was measured using mercury sphygmomanometer with the patient in the sitting position after 5 minutes of rest. Three readings separated by 2 minutes were taken and the average was used for calculation. Mean arterial blood pressure [diastolic blood pressure $+1 / 3$ (systolic blood pressure - diastolic blood pressure)] and Body Mass Index (BMI) were recorded. The glomerular filtration rate was estimated by Modification of Diet in Renal Disease Abbreviated Equation (MDRD): $\left[\mathrm{GFR}=186 \times(\text { serum } \mathrm{Cr})^{-1.154} \times(\text { age })^{-0.203} \times(0.742\right.$ if female $) \times(1.210$ if African American)]. Glycemic control was assessed by glycosylated hemoglobin (HbA1c). Proteinuria was measured by albumin dipsticks, urinary microalbumin kits and protein/creatinine ( $\mathrm{Pr} / \mathrm{Cr})$ ratio in morning urine sample. Pelvic and abdominal ultrasonographic examination was done for all subjects.

Blood and urine samples for the estimation of serum and urine TNF- $\alpha$ were drawn from each subject in the morning (between 8 and $11 \mathrm{am}$ ). Blood samples were collected in sterile tubes, centrifuged and the supernatant stored with urine samples at $-70^{\circ} \mathrm{C}$ until assayed by immunoenzymetric assay for the in-vitro quantitative measurement of human TNF- $\alpha$ using BioSource TNF- $\alpha$ EASIA Kit. Estimation of serum high sensitivity C-reactive protein (hs-CRP) was done for all subjects by enzyme-linked immunosorbent assay (ELISA). 
Table 1: Comparison between all groups as regards studied variables

\begin{tabular}{lllllll}
\hline & $\begin{array}{l}\text { Control } \\
(\text { mean } \pm \text { SD) }\end{array}$ & $\begin{array}{l}\text { Group I } \\
(\text { mean } \pm \text { SD) }\end{array}$ & $\begin{array}{l}\text { Group II } \\
(\text { mean } \pm \text { SD) }\end{array}$ & $\begin{array}{l}\text { Group III } \\
(\text { mean } \pm \text { SD) }\end{array}$ & $\begin{array}{l}\text { Group IV } \\
(\text { mean } \pm \text { SD) }\end{array}$ & P value \\
\hline Duration of diabetes (years) & & $8.9 \pm 2.8$ & $13.9 \pm 3.8$ & $18.1 \pm 4.5$ & $7.9 \pm 2.6$ & 0.00 \\
MABP (mmHg) & $89.7 \pm 4.1$ & $100.6 \pm 11.8$ & $105 \pm 14.3$ & $106.6 \pm 11.7$ & $100.2 \pm 9.8$ & 0.00 \\
BMI & $29.2 \pm 3.6$ & $28.4 \pm 4.8$ & $27.8 \pm 3.3$ & $29.3 \pm 4.2$ & $29.3 \pm 4.4$ & 0.58 \\
eGFR (ml/min/1.73m²) & - & $102 \pm 10.1$ & $102.1 \pm 10.7$ & $19.7 \pm 13.4$ & $103 \pm 14$ & - \\
Hb A1C & - & $8.1 \pm 1.1$ & $7.4 \pm 1.4$ & $7.7 \pm 1.4$ & $7.3 \pm 1.1$ & 0.24 \\
Pr/Cr & - & - & $2.11 \pm 1.44$ & $2.26 \pm 1.39$ & $0.07 \pm 0.04$ & 0.00 \\
Serum TNF- $\alpha(\mathrm{pg} / \mathrm{ml})$ & $36.3 \pm 6.7$ & $478.5 \pm 117.1$ & $686.7 \pm 177.6$ & $467 \pm 293$ & $244.3 \pm 93.7$ & 0.00 \\
Urine TNF- $\alpha(\mathrm{pg} / \mathrm{mg})$ & $33.3 \pm 11.9$ & $420.8 \pm 141.5$ & $570 \pm 126.4$ & $472.7 \pm 247.3$ & $225 \pm 79.46$ & 0.00 \\
hs-CRP $(\mathrm{mg} / \mathrm{l})$ & $0.6 \pm 0.4$ & $4.4 \pm 1.1$ & $7.2 \pm 1.6$ & $6.2 \pm 2.4$ & $2.9 \pm 1.2$ & 0.00 \\
& & & & & & \\
\hline
\end{tabular}

MABP: mean arterial blood pressure; BMI: body mass index; eGFR: estimated glomerular filteration rate; HbA1C: glycated hemoglobin C; Pr/Cr: protein creatinine ratio; serum TNF- $\alpha$ : serum tumour necrosis factor alpha; urine TNF- $\alpha$ : urine tumour necrosis factor alpha; hs-CRP; high sensitivity C-reactive protein

Correlation between serum and urine TNF- $\alpha$ and serum CRP was done with the duration of diabetes, mean arterial blood pressure, HbA1c, degree of proteinuria, as well as estimated GFR. Data were analyzed using Statistical Package for Social Sciences (SPSS) software computer program version 15 . Mean \pm standard deviation and frequencies were used to describe quantitative and qualitative data respectively. Independent Student t-test was used for comparison of quantitative variables between groups. One-way ANOVA test was used for comparison of quantitative variables among more than two independent groups. Chi-square test was used for comparison of distribution of qualitative variables among different groups. Correlation between continuous variables was performed using Pearson correlation coefficient. $\mathrm{P}$ value $\leq 0.05$ was considered significant.

Forward stepwise multiple regression analysis was performed to determine the independent association between relevant patient characteristics and clinical parameters as potential predictor variables (age, gender, duration of diabetes, BMI, MABP, HbA1C, serum TNF- $\alpha$, urine TNF- $\alpha$, hs-CRP) and estimated GFR and $\mathrm{Pr} / \mathrm{Cr}$ ratio as the dependant variable.

\section{Results}

Baseline characteristics of the studied groups are shown (Table 1). Levels of serum TNF- $\alpha$, urine TNF- $\alpha$ and hs-CRP were significantly different between study groups (Figures 1-2). Serum TNF- $\alpha$ levels were significantly and positively correlated to the duration of diabetes and HbA1C levels. Urine TNF- $\alpha$ levels were significantly and positively correlated to the duration of diabetes but not to HbA1C levels.

Scatter blots revealed a positive correlation between serum TNF- $\alpha$, urine TNF- $\alpha$, hs-CRP and $\mathrm{Pr} / \mathrm{Cr}$ ratio (Figures 3-5). After forward stepwise multiple regression analysis, serum TNF- $\alpha$ and urine TNF- $\alpha$ remained as independent predictors of $\mathrm{Pr} / \mathrm{Cr}$ among patients in group-II ( $\mathrm{R} 2=0.46$ and 0.32 respectively, $\mathrm{P}<0.05)$ and group-III ( $\mathrm{R} 2=0.36$ and 0.31 respectively, $\mathrm{P}<0.05$ ).

\section{Discussion}

Diabetic nephropathy (DN) is the principal cause of ESRD in the Western world, with renal disease as a major cause of morbidity and mortality in the diabetic population. It has been estimated that type 2 diabetes now contributes to at least $50 \%$ of patients who have diabetes in end-stage renal failure programs [3]. There is growing evidence that activated innate immunity and inflammation are relevant factors in the pathogenesis of diabetes; with convincing data that type-2 diabetes includes an inflammatory component [5]. Regarding diabetic complications, experimental and clinical studies have demonstrated that DN exhibits signs of inflammation, with proinflammatory cytokines being suggested as important factors in the development of renal injury [6]. Several studies have reported that serum levels of inflammatory markers are greater in patients with diabetes with increased urinary albumin excretion (UAE) with respect to normoalbuminuric subjects. Also there was a significant relationship between serum TNF- $\alpha$ level and urinary protein excretion in patients with type-2 
Figure 1: Comparison of serumTNF- $\alpha$ and urine TNF- $\alpha$ between study groups $(\mathrm{P}=0.00)$

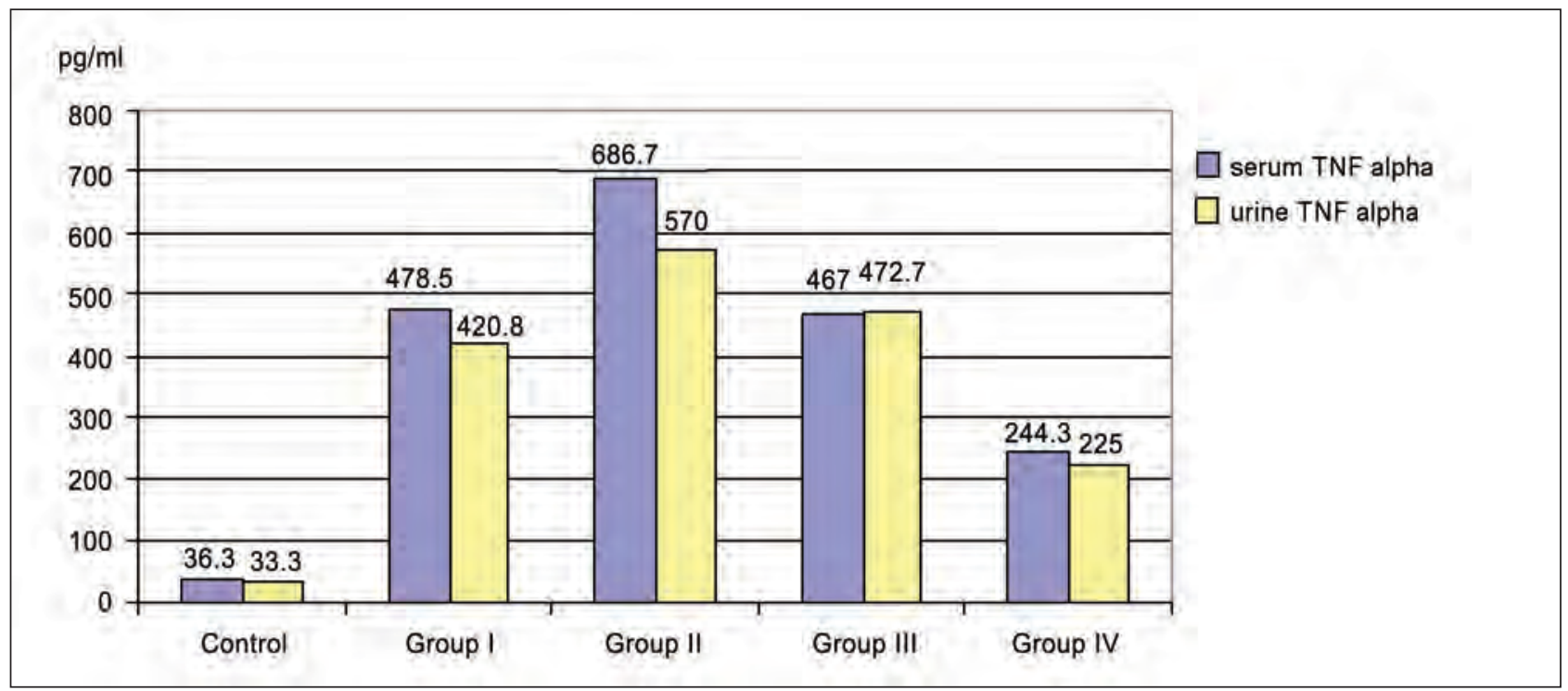

diabetes with overt nephropathy and renal insufficiency [3]. Studies on animal models of DN have shown that renal expression of TNF- $\alpha$ is increased compared to kidneys of non-diabetic animals [13]. Preliminary clinical reports have found a significant relationship between the urinary excretion of this cytokine and UAE in type- 2 diabetic patients. Also serum TNF- $\alpha$ has been reported to be elevated in diabetic subjects, and even in patients with only impaired glucose tolerance, compared to healthy individuals [11]. However, clinical studies analyzing the serum and urinary excretion of TNF- $\alpha$ in diabetic patients in relation to proteinuria are scarce. Our study highlights the possible role of TNF- $\alpha$ in the development and progression of renal injury in diabetic patients, and demonstrates that serum and urinary concentrations of this cytokine are enhanced in diabetic subjects, increasing as renal damage progresses. The availability of highly sensitive assays resulted in that hs-CRP has become an exquisite marker of chronic subclinical inflammation. In our study, hs-CRP levels in type-2 diabetic patients with microalbuminuria and overt proteinuria were significantly greater than in diabetic patients without albuminurea and healthy individuals. Furthermore, there was a positive correlation between serum and urine TNF- $\alpha$ and hs-CRP level and urinary protein excretion. These results are in agreement with the results of Navarro et al, who compared 65 type-2 diabetic patients and 21 healthy controls and found that serum and urine TNF- $\alpha$ and hs-CRP were significantly higher in diabetic than in control subjects [3]. We also observed that the mean values of serum and urine TNF- $\alpha$ and hs-CRP are higher in diabetics with proteinuria than those without albuminuria. The results of Moriwaki et al support our findings, as he observed that serum TNF- $\alpha$ levels in diabetic patients with microalbuminuria were significantly increased as compared to those without albuminuria [14]. We also observed that levels of these inflammatory cytokines were higher in diabetics with macroalbuminuria compared to those with microalbuminuria. This suggests that levels of serum and urine TNF- $\alpha$ and hs-CRP increase when proteinuria increases and nephropathy progresses. These results are in agreement with those of Navarro et al, who observed significant differences in serum and urine TNF- $\alpha$ and hs-CRP levels between patients with microalbuminuria and patients with macroalbuminuria, being higher among patients with macroalbuminuria [11].

We found significant association between serum, but not urinary, TNF- $\alpha$ and $\mathrm{HbA} 1 \mathrm{C}$ level. The reverse was found in the studies of Navarro et al, who attributed their findings to the relation between metabolic control and kidney production of this cytokine $[3,11]$. This is conceivable, but metabolic control could also affect the severity of the disease and its progression and hence systemic production of this cytokine, which could explain the correlation between metabolic control and serum TNF- $\alpha$ in our results. The observations of Chen et al support our finding, as he observed that serum TNF- $\alpha$ level was related to glycemic control (HbA1C). They proposed that hyperglycemia can induce elevated serum TNF- $\alpha$ levels through oxidative stress pathway [15]. Further studies are required to confirm these associations. The relationship between serum TNF- $\alpha$ and BMI is a matter of debate. 
Figure 2: Comparison of hs-CRP between studied groups $(\mathbf{P}=\mathbf{0 . 0 0 )}$

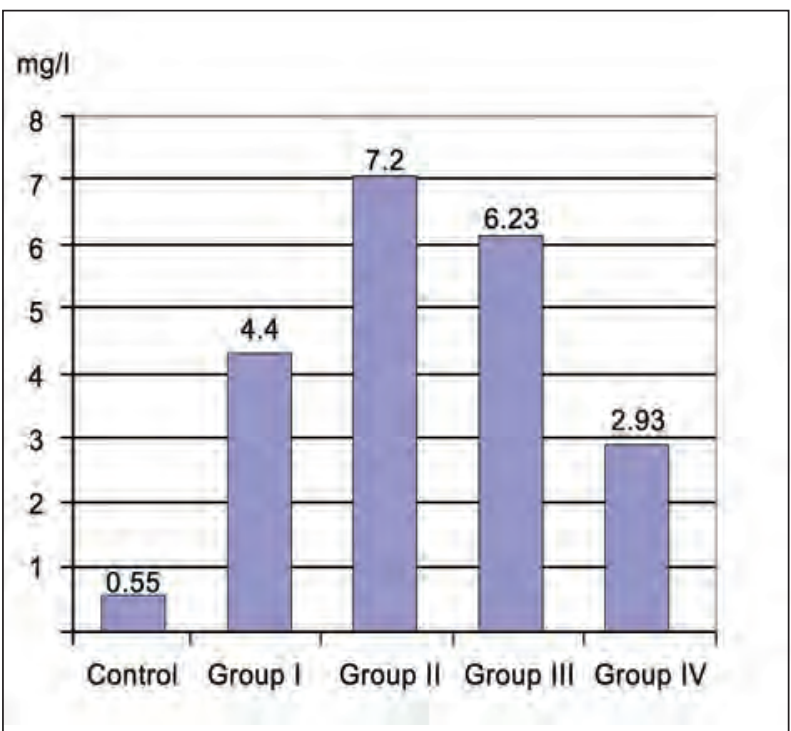

Some authors showed no relation between the two while others did. In a study conducted by Lele et al on 35 diabetic subjects and 50 healthy controls, no association was found between serum TNF- $\alpha$ and BMI [16]. On the contrary, Navarro et al found significant correlation between serum TNF- $\alpha$ and BMI [11]. They explained that serum levels of TNF- $\alpha$ are essentially determined by the systemic production of this adipocytokine by adipose tissue, unrelated to metabolic factors such as HbA1C. This difference in results could be attributed to the fact that Lele et al conducted his study on Asian Indians. He reported that Asian Indians have unique biochemical and hormone features which may be considered as additional features to the so called 'Asian Indian Phenotype' [16]. Our results concur with those of of Lele et al, as we found no significant association between serum TNF- $\alpha$ and BMI. This may also have resulted from genetic and racial differences.

Our results showed that serum and urine TNF- $\alpha$ were independently and significantly associated with $\mathrm{Pr} / \mathrm{Cr}$ in Group-II and Group-III. These results are supported by those of Navarro et al who also found that serum and urine TNF- $\alpha$ were independent of and significantly associated with UAE [11].

\section{Conclusion}

Serum and urine TNF- $\alpha$ and hs-CRP levels are elevated in type-2 diabetic patients and their levels correlate with markers of glomerular damage. This suggests a significant role for TNF- $\alpha$ in the pathogenesis and progression of renal injury in diabetes mellitus.
Figure 3: Correlation between serum TNF- $\alpha$ level and $\mathrm{Pr} / \mathrm{Cr}$ ratio

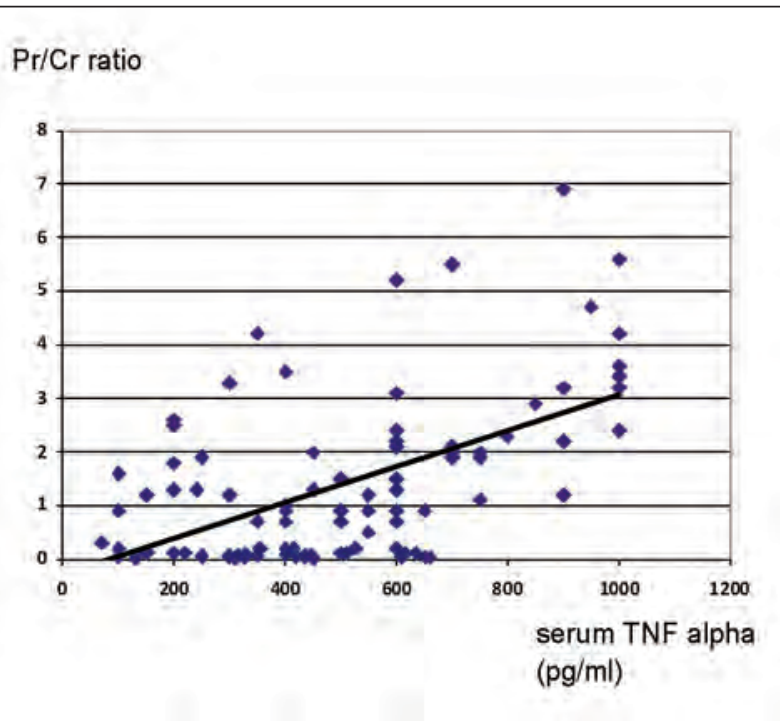

\section{References}

1. Caramori ML, Mauer M. Diabetes and nephropathy. Curr Opin Nephrol Hypertens. 2003 May;12(3):273-82.

2. Buraczynska K, Koziol-Montewka M, Majdan M, Tokarz A, Ksiazek A. Genetic determination of TNF and myeloperoxidase production in dialyzed patients with diabetic nephropathy. Ren Fail. 2004 Nov;26(6):633-9.

3. Navarro JF, Mora C, Maca M, Garca J. Inflammatory parameters are independently associated with urinary albumin in type 2 diabetes mellitus. Am J Kidney Dis. 2003 Jul;42(1):53-61.

4. Sassy-Prigent C, Heudes D, Mandet C, Bélair MF, Michel O, Perdereau B, Bariéty J, Bruneval P. Early glomerular macrophage recruitment in streptozotocininduced diabetic rats. Diabetes. 2000 Mar;49(3):466-75.

5. Crook M. Type 2 diabetes mellitus: a disease of the innate immune system? An update. Diabet Med. 2004 Mar;21(3):203-7.

6. Mora C, Navarro JF. The role of inflammation as a pathogenic factor in the development of renal disease in diabetes. Curr Diab Rep. 2005 Dec;5(6):399-401

7. Kalantarinia K, Awad AS, Siragy HM. Urinary and renal interstitial concentrations of TNF- $\alpha$ increase prior to the rise in albuminuria in diabetic rats. Kidney Int. 2003 Oct;64(4):1208-13.

8. Bertani T, Abbate M, Zoja C, Corna D, Perico N, Ghezzi P, Remuzzi G. Tumor necrosis factor induces 
Figure 4: Correlation between urine TNF- $\alpha$ level and $\mathrm{Pr} / \mathrm{Cr}$ ratio

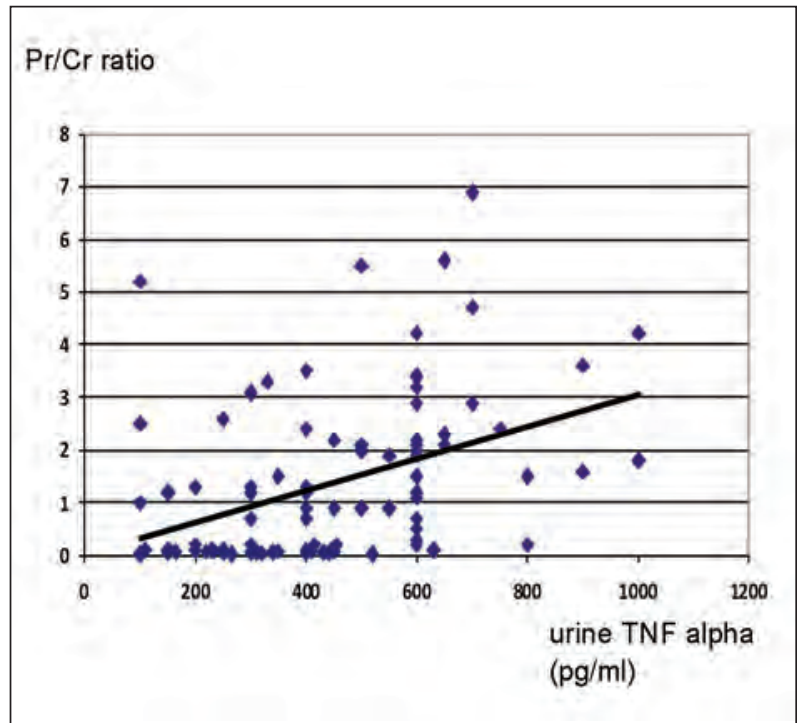

glomerular damage in the rabbit. Am J Pathol. 1989 Feb;134(2):419-30.

9. Navarro JF, Mora-Fernández C. The role of TNF-alpha in diabetic nephropathy: pathogenic and therapeutic implications. Cytokine Growth Factor Rev. 2006 Dec;17(6):441-50.

10. Hasegawa G, Nakano K, Sawada M, Uno K, Shibayama Y, Ienaga K, Kondo M. Possible role of tumor necrosis factor and interleukin-1 in the development of diabetic nephropathy. Kidney Int. 1991 Dec;40(6):100712

11. Navarro JF, Mora C, Muros M, García J. Urinary tumour necrosis factor-alpha excretion independently correlates with clinical markers of glomerular and tubulointerstitial injury in type 2 diabetic patients. Nephrol Dial Transplant. 2006 Dec;21(12):3428-34.

12. Moriwaki Y, Inokuchi T, Yamamoto A, Ka T, Tsutsumi Z, Takahashi S, Yamamoto T. Effect of TNF-alpha inhibition on urinary albumin excretion in experimental diabetic rats. Acta Diabetol. 2007 Dec;44(4):215-8.
Figure 5: Correlation between hs-CRP level and Pr/Cr ratio among patients

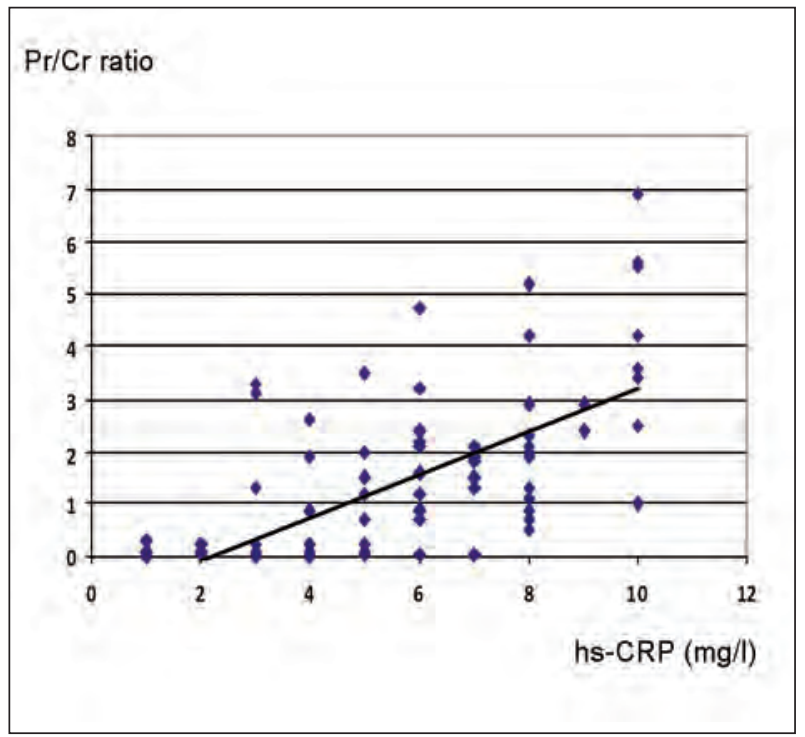

13. Navarro JF, Milena FJ, Mora C, León C, Claverie F, Flores C, García J. Tumor necrosis factor-alpha gene expression in diabetic nephropathy: relationship with urinary albumin excretion and effect of angiotensinconverting enzyme inhibition. Kidney Int Suppl. 2005 Dec;(99):S98-102.

14. Moriwaki Y, Yamamoto T, Shibutani Y, Aoki E, Tsutsumi Z, Takahashi S, Okamura H, Koga M, Fukuchi M, Hada T. Elevated levels of interleukin-18 and tumor necrosis factor-alpha in serum of patients with type 2 diabetes mellitus: relationship with diabetic nephropathy. Metabolism. 2003 May;52(5):605-8.

15. Chen H, Ren A, Hu S, Mo W, Xin X, Jia W. The significance of tumor necrosis factor-alpha in newly diagnosed type 2 diabetic patients by transient intensive insulin treatment. Diabetes Res Clin Pract. 2007 Mar;75(3):327-32.

16. Lele RD, Joshi SR, Gupte A. Association of adipocytokines (leptin, adiponectin TNF-alpha), insulin and proinsulin with diabetes: the Mumbai Obesity Project [MOP]. J Assoc Physicians India. 2006 Sep;54:689-96. 